\title{
Constraint programming for wireless sensor networks
}

\author{
Farshid Hassani Bijarbooneh ${ }^{1}$
}

Published online: 8 October 2015

(C) Springer Science+Business Media New York 2015

\begin{abstract}
In recent years, wireless sensor networks (WSNs) have grown rapidly and have had a substantial impact in many applications. A WSN is a network that consists of interconnected autonomous nodes that monitor physical and environmental conditions, such as temperature, humidity, pollution, etc. If required, nodes in a WSN can perform actions to affect the environment. WSNs present an interesting and challenging field of research due to the distributed nature of the network and the limited resources of the nodes. It is necessary for a node in a WSN to be small to enable easy deployment in an environment and consume as little energy as possible to prolong its battery lifetime. There are many challenges in WSNs, such as programming a large number of nodes, designing communication protocols, achieving energy efficiency, respecting limited bandwidth, and operating with limited memory. WSNs are further constrained due to the deployment of the nodes in indoor and outdoor environments and obstacles in the environment. In this dissertation, we study some of the fundamental optimisation problems related to the programming, coverage, mobility, data collection, and data loss of WSNs, modelled as standalone optimisation problems or as optimisation problems integrated with protocol design. Our proposed solution methods come from various fields of research including constraint programming, integer linear programming, heuristic-based algorithms, and data inference techniques.
\end{abstract}

School: Uppsala University

\section{Supervisors:}

Justin Pearson

Pierre Flener

Edith Ngai

Graduated: Friday, March 13, 2015

Link to full text: http://uu.diva-portal.org/smash/get/diva2:778823/FULLTEXT01.pdf

Farshid Hassani Bijarbooneh

farshid.hassani@gmail.com

1 Uppsala University, Uppsala, Sweden 